
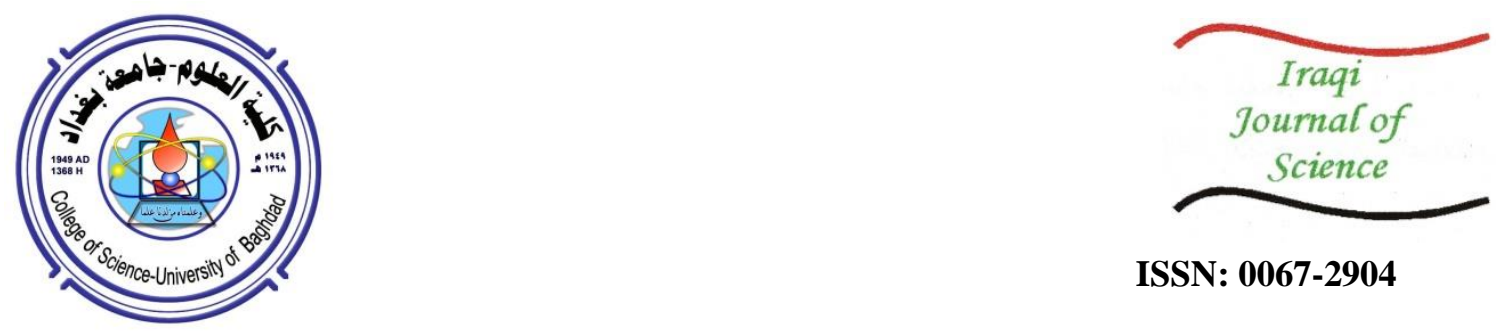

ISSN: 0067-2904

\title{
On Closed Quasi Principally Injective Acts over Monoids
}

\author{
Shaymaa Amer Abdul-Kareem ${ }^{1 *}$, Ahmed Amer Abdulkareem2, Yusra Amer \\ Abdul-Kareem ${ }^{3}$ \\ ${ }^{1}$ Department of Mathematics, College of Basic Education, Mustansiriyah University, Baghdad, Iraq \\ ${ }^{2}$ Ministry of trade, Department of Follow-up and Monitoring Electronic \\ ${ }^{3}$ Ministry of trade, Department of Foreign and Economic Relations
}

Received: 8/6/2020

Accepted: 5/9/2020

\begin{abstract}
The concept of closed quasi principally injective acts over monoids is introduced, which signifies a generalization for the quasi principally injective as well as for the closed quasi injective acts. Characterization of this concept is intended to show the behavior of a closed quasi principally injective property. At the same time, some properties of closed quasi principally injective acts are examined in terms of their endomorphism monoid. Also, the characterization of a closed self-principally injective monoid is given in terms of its annihilator. The relationship between the following concepts is also studied; closed quasi principally injective acts over monoids, Hopfian, co Hopfian, and directly finite property. Ultimately, based on the results obtained, the conditions on subacts to inherit a closed quasi principally injective property were shown. Part of this paper was dedicated to studying the relationship between the classes of closed quasi principally injective acts with some generalizations of injectivity. Conclusions and future remarks of this work are given.
\end{abstract}

Keywords: Quasi Principally Injective Acts, Closed Quasi Principally Injective Acts, Closed Self Principally Injective Monoid, Continuous Acts, Extending Acts, Generalization of Quasi Principally injective acts. AMS Subject Classification: 20M30, 20M99, 08B30.

$$
\begin{aligned}
& \text { حول الانظمة شبه الرئيسية الاغماريـة المغلقة على اشباه الزمر الاحادية } \\
& \text { شيماء عامر عبد الكريم1" ، احمد عامر عبد الكريم² ، يسرى عامر عبد الكريم3 }
\end{aligned}
$$

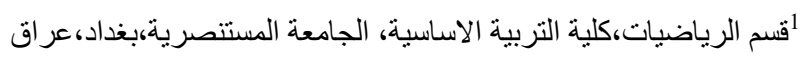

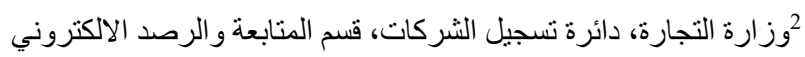

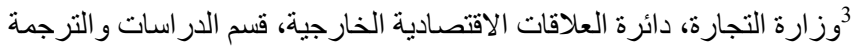

*Email: Shaymma_amer.edbs@uomustansiriyah.edu.iq 


\section{الخلاصة}

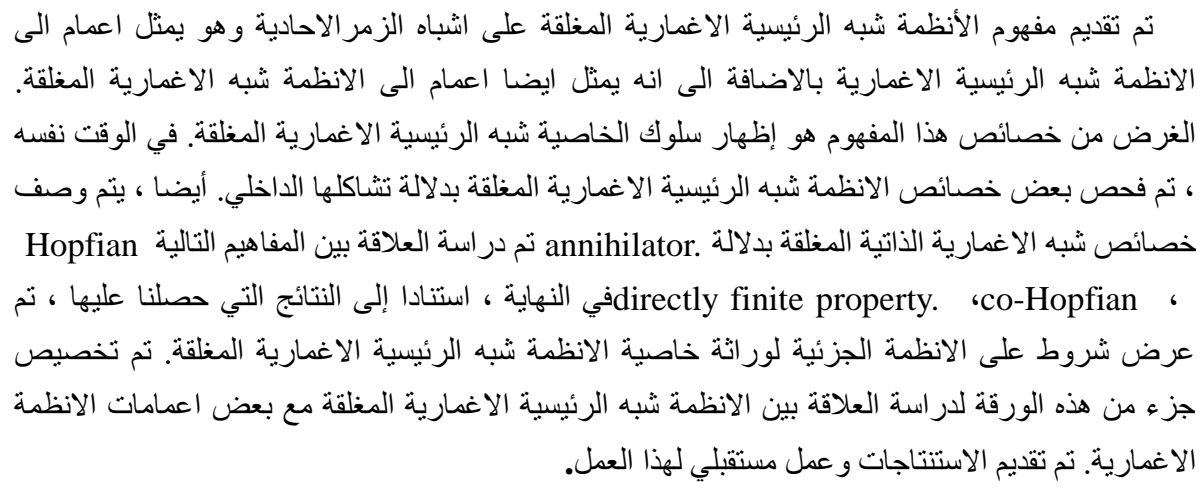

\section{Introduction}

For any given mathematical structure on a set, the collection of structure-preserving maps on the set to itself is an example of an abstract algebraic "object", referred to as a semigroup. Thereby, semigroups pervade mathematics. On the other hand, given an abstractly defined semigroup, when can it be represented as a semigroup of maps of a mathematical structure? The answer is represented by actions. In mathematics, an action of a semigroup on a set is an operation that associates each element of the semigroup with a transformation on the set. It is familiar that, from an algebraic perspective, an action for the semigroup is a generalization of the notion of group action in group theory, and a major special case is a monoid action or act, in which the semigroup is a monoid and the identity element of the monoid acts as the identity transformation of a set. It is recognized that the theory of monoids and acts is a generalization of the theory of rings and modules, which has a number of direct applications in theoretical computer science, theory of differential equations and functional analysis, etc. [1].

Throughout this work, every right $\mathrm{S}$-act $\mathrm{M}$ is a unitary S-act (contains identity element), with zero element $\Theta$ represented by $M_{S}$, and $S$ is a monoid with zero elements 0 . Let $M_{S}$ refers to a right $S$-act with zero where it is a non-empty set with a function $\mathrm{f}: \mathrm{M} \times \mathrm{S} \rightarrow \mathrm{M},(\mathrm{m}, \mathrm{s}) \mapsto \mathrm{ms}$ such that the following properties hold: (1) $\mathrm{m} \cdot 1=\mathrm{m}(\mathbf{2}) \mathrm{m}(\mathrm{st})=(\mathrm{ms}) \mathrm{t}$, for all $\mathrm{m} \in \mathrm{M}$ and $\mathrm{s}, \mathrm{t} \in \mathrm{S}$, where 1 denotes the identity element of S. For other basic definitions, theorems, lemmas, corollaries, results and notations for S-acts, annihilators, homomorphism, endomorphism, monomorphism, epimorphism, isomorphism ...etc. we refer to [2, 3 and 4].

It is possible to find an S-act in different names such as S-acts, S-sets, S-operands, S-polygons, transition acts, and S-automata [2].We will freely make use of the standard notations, terminologies as well as results of $[1,5,6,7,8$ and 9$]$.

Let $A_{S}$ and $M_{S}$ be two S-acts. $A_{S}$ is referred to as an M-injective in case of an S-monomorphism $\alpha$ : $\mathrm{N} \rightarrow \mathrm{M}_{\mathrm{S}}$ where $\mathrm{N}$ is a subact of $\mathrm{M}_{\mathrm{S}}$ and every S-homomorphism $\beta: \mathrm{N} \rightarrow \mathrm{A}_{S}$, can be extended to an S-homomorphism $\sigma: \mathrm{M}_{\mathrm{S}} \rightarrow \mathrm{A}_{\mathrm{S}}[10]$.

An S-act $A_{S}$ is an injective if it is an M-injective for all S-acts $M_{S}$. An S-act $A_{S}$ is quasi injective if and only if it is an A-injective. Quasi injective S-acts were studied by Lopez and Luedeman [11]. In [1], the author developed the concept presented by Lopez to C-quasi injective act. An S-act $\mathrm{N}_{\mathrm{S}}$ is called closed M-injective (for short C-M-injective) if for any homomorphism from a closed subact of S-act $\mathrm{M}_{\mathrm{S}}$ to $\mathrm{N}_{\mathrm{S}}$ can be extended to a homomorphism from $\mathrm{M}_{\mathrm{S}}$ to $\mathrm{N}_{\mathrm{S}}$ [1]. An $\mathrm{S}$-act $\mathrm{N}_{\mathrm{S}}$ is referred to as a C-quasi injective if $\mathrm{N}_{S}$ is $\mathrm{C}$-N-injective. In a similar way, a monoid $\mathrm{S}$ is called the right $\mathrm{C}$-self-injective if it is C-S-injective. Besides, the author continues to develop and generalize the concept of the quasi injective act introduced by Lopez to quasi principally injective act. An S-act $\mathrm{N}_{\mathrm{S}}$ is 
called M-principally injective if every S-homomorphism from M-cyclic subact of S-act $\mathrm{M}_{\mathrm{S}}$ into $\mathrm{N}_{\mathrm{S}}$ can be extended to an $\mathrm{S}$-homomorphism from $\mathrm{M}_{\mathrm{S}}$ into $\mathrm{N}_{\mathrm{S}}$ (for short $\mathrm{N}_{\mathrm{S}}$ is M-P-injective) [8]. Equally, an $\mathrm{S}$-act $\mathrm{M}_{\mathrm{S}}$ is referred to as quasi-principally injective if it is M-P-injective [8]. In [12], Al-Bahrani and Rahman introduced a generalization of Rickart Modules to y-closed Rickart Modules. Because the S-act theory is a generalization of module theory, we introduced the generalization of quasi principally injective acts over monoids to closed quasi principally injective acts over monoids.

This paper aims to introduce and study the concept of closed quasi principally injective acts by examining their structure and properties. The importance of this concept is attributed to two points: Firstly, it represents a generalization of closed quasi injective acts and, secondly, it signifies a generalization of quasi principally injective acts. In addition, we characterized the behavior of the property that is considered under well-known constructions such as the product, coproduct, and direct sum. This article is divided into three sections. Section two is devoted to introduce and investigate a new kind of generalization of quasi principally injective S-acts, namely closed quasi principally injective act over monoids. Certain classes of subacts which inherit the property of closed quasi principally injective acts were considered. Also, the characterizations of this new class of S-acts were investigated. An example was given to demonstrate closed quasi principally injective acts over monoids. Some known results on closed quasi principally injective for general modules were generalized to S-acts.

For future work, one can consider the subact that is closed and finitely M-generated.

\section{Results}

Definition 2.1: [8] Let $\mathrm{M}_{S}$ and $\mathrm{N}_{\mathrm{S}}$ be two S-acts. An S-act $\mathrm{N}_{\mathrm{S}}$ is called M-principally injective if every S-homomorphism of M-cyclic subact of $\mathrm{M}_{\mathrm{S}}$ into $\mathrm{N}_{\mathrm{S}}$ can be extended to an S-homomorphism from $\mathrm{M}_{\mathrm{S}}$ into $\mathrm{N}_{\mathrm{S}}$ (if this is the case, we write $\mathrm{N}_{\mathrm{S}}$ as M-P-injective).

Definition2.2: [1] Let $M_{S}$ and $N_{S}$ be two S-acts, $N_{S}$ is called closed M-injective (for short C-M injective) if any homomorphism of a closed subact of $\mathrm{M}_{\mathrm{S}}$ to $\mathrm{N}_{\mathrm{S}}$ can be extended to homomorphism from $\mathrm{M}_{\mathrm{S}}$ to $\mathrm{N}_{\mathrm{S}}$. An S-act $\mathrm{N}_{\mathrm{S}}$ is called closed quasi injective if $\mathrm{N}_{\mathrm{S}}$ is $\mathrm{C}-\mathrm{N}$-injective. A monoid $\mathrm{S}$ is called right closed self-injective if it is C-S-injective.

Definition2.3: An S-act $\mathrm{N}_{S}$ is called closed M-principally injective (for short, C-M-P-injective) if every S-homomorphism of closed M-cyclic subact of $\mathrm{M}_{\mathrm{S}}$ to $\mathrm{N}_{\mathrm{S}}$ extends to $\mathrm{S}$-homomorphism from $\mathrm{M}_{\mathrm{S}}$ to $\mathrm{N}_{\mathrm{S}}$. Meanwhile, an $\mathrm{S}$-act $\mathrm{M}_{\mathrm{S}}$ is called closed quasi principally injective (for short, C-QP-injective) if it is closed M-principally injective. Similarly, a monoid $\mathrm{S}$ is called closed self principally injective monoid (for short, C-self-P-injective) in case that $S_{S}$ is closed quasi principally injective.

\section{Remarks and Example2.4}

(1) Recall that an S-act $M_{S}$ is called quasi-principally injective if it is M-P-injective, that is every S-homomorphism from M-cyclic subact of $M_{S}$ to $M_{S}$ can be extended to S-endomorphism of $M_{S}$. Accordingly, we mention that $\mathrm{M}_{\mathrm{S}}$ is QP-injective [8]. For this reason, every QP-injective is $\mathrm{C}$-QP-injective, but the converse is not true in general. For example, $\mathrm{Z}$ with usual multiplication monoid as $\mathrm{Z}$-act is $\mathrm{C}$-QP-injective which cannot be called quasi principally injective act.

(2) Obviously, definition2.3 is up to isomorphism. This means that every object may be replaced by an isomorphic object.

Recall that $\alpha$ is an $S$-homomorphism if it is a mapping (i.e. $\alpha: A_{S} \rightarrow B_{S}$ ) from $S$-act $A_{S}$ into $S$-act $B_{S}$ such that for any $\mathrm{a} \in \mathrm{A}_{\mathrm{S}}$ and $\mathrm{s} \in \mathrm{S}, \alpha(\mathrm{as})=\alpha(\mathrm{a}) \mathrm{s}$. The usual meanings of monomorphism, epimorphism, and isomorphism are also satisfied [7]. Besides, an S-homomorphism $f: M_{S} \rightarrow M_{S}$ is called an endomorphism of $\mathrm{M}_{\mathrm{S}}$, where $\mathrm{M}_{\mathrm{S}}$ is $\mathrm{S}$-act. 
Definition 2.5: An endomorphism $f \in E n d(M)$ is called a closed homomorphism if $f(M)$ is a closed subact of $\mathrm{M}_{\mathrm{S}}$.

The following theorem illustrates the characterization of C-quasi principally injective act (for definition of annihilators see definition (1.1.27) in [7]).

Theorem 2.6: Let $\mathrm{M}_{\mathrm{S}}$ be an $\mathrm{S}$-act and $\mathrm{T}=\mathrm{End}(\mathrm{M})$. Then the following conditions are equivalent:

(1) $\mathrm{M}_{\mathrm{S}}$ is C-M-P-injective.

(2) $\ell_{\mathrm{T}}(\mathrm{ker} \alpha)=\mathrm{T} \alpha$ For every closed homomorphism $\alpha \in \mathrm{T}$.

(3) If $\mathrm{f:} \alpha(\mathrm{M}) \rightarrow \mathrm{M}_{\mathrm{S}}$ is a homomorphism, then $\mathrm{f} \alpha \in \mathrm{T} \alpha$ for closed homomorphism $\alpha \in \mathrm{T}$.

(4) $\operatorname{Ker} \alpha \subseteq \operatorname{Ker} \beta$ implies that $\mathrm{T} \beta \subseteq \mathrm{T} \alpha$ for any $\alpha, \beta \in \mathrm{T}$, where $\alpha$ is a closed homomorphism.

(5) $\ell_{\mathrm{T}}[(\beta(\mathrm{M}) \times \beta(\mathrm{M})) \cap \mathrm{Ker} \alpha]=\ell_{\mathrm{T}}(\beta(\mathrm{M}) \times \beta(\mathrm{M})) \mathrm{UT} \alpha$, for each $\alpha, \beta \in \mathrm{T}$ where $(\alpha \beta)$ is a closed homomorphism and $\mathrm{T}$ is right cancellative.

Proof: $(1 \rightarrow 2)$ Let $\beta \in \mathrm{T} \alpha$ be a closed homomorphism. For any $\sigma \in \mathrm{T}$, we have then that $\beta=\sigma \alpha$, so $\operatorname{Ker}(\alpha) \subseteq \operatorname{Ker}(\sigma \alpha)$. For each $\mathrm{s}, \mathrm{t} \in \mathrm{S}$ with $\mathrm{ms}=\mathrm{mt}$, we have $\alpha(\mathrm{ms})=\alpha(\mathrm{mt})$ and then $\sigma \alpha(\mathrm{ms})=$ $\sigma \alpha(\mathrm{mt})$. This implies that $\beta(\mathrm{ms})=\beta(\mathrm{mt})$, so $\beta \in \ell_{\mathrm{T}}(\operatorname{Ker} \sigma \alpha) \subseteq \ell_{\mathrm{T}}(\operatorname{Ker} \alpha)$. Conversely, let $\beta \in$ $\ell_{\mathrm{T}}(\operatorname{Ker} \alpha)$, then define $\sigma: \alpha\left(\mathrm{M}_{\mathrm{S}}\right) \rightarrow \mathrm{M}_{\mathrm{S}}$ by $\sigma(\alpha(\mathrm{m}))=\beta(\mathrm{m})$ for some $\mathrm{m}_{\mathrm{S}} \mathrm{M}_{\mathrm{S}}$. It is clear that $\sigma$ is a well-defined and S-homomorphism with Ker $\alpha \subseteq \operatorname{Ker} \beta$. In fact, if $\alpha(\mathrm{ms})=\alpha(\mathrm{mt})$, then $\sigma(\alpha(\mathrm{ms}))=$ $\sigma(\alpha(\mathrm{mt}))$.Thus, $\beta(\mathrm{ms})=\beta(\mathrm{mt})$, which means that $\sigma$ is well-defined. From this, it is easy to see that $\sigma$ is S-homomorphism. Let $\alpha^{\prime}: \mathrm{M}_{\mathrm{S}} \rightarrow \alpha\left(\mathrm{M}_{\mathrm{S}}\right)$, and $\beta^{\prime}: \mathrm{M}_{\mathrm{S}} \rightarrow \beta\left(\mathrm{M}_{\mathrm{S}}\right)$ be S-epimorphisms induced by $\alpha$ and $\beta$, respectively. Let $i_{1}: \alpha\left(M_{S}\right) \rightarrow M_{S}, i_{2}: \beta\left(M_{S}\right) \rightarrow M_{S}$ be the inclusion maps. Since $\alpha /$ is S-epimorphism, so there is an S-homomorphism $\varphi: \alpha\left(\mathrm{M}_{\mathrm{S}}\right) \rightarrow \beta\left(\mathrm{M}_{\mathrm{S}}\right)$ such that $\varphi \alpha^{/}=\beta^{\prime}, \forall \mathrm{m} \in \mathrm{M}_{\mathrm{S}}$. Let $\alpha(\mathrm{m}) \in \alpha\left(\mathrm{M}_{\mathrm{S}}\right)$. Since $\alpha^{\prime}$ is epimorphism, so there exists $\mathrm{x} \in \mathrm{M}_{\mathrm{S}}$ such that $\alpha^{\prime}(\mathrm{x})=\alpha(\mathrm{m})$, so $\varphi(\alpha(\mathrm{m}))=\beta(\mathrm{x})$, where $\alpha^{\prime}(\mathrm{x})=\alpha(\mathrm{m})$ and $\beta^{\prime}(\mathrm{x})=\beta(\mathrm{x})$. Now, $\varphi$ is well-defined. If $\alpha\left(\mathrm{m}_{1}\right)=\alpha\left(\mathrm{m}_{2}\right)$ with $\alpha^{\prime}\left(\mathrm{x}_{1}\right)=\alpha\left(\mathrm{m}_{1}\right)$ and $\alpha^{\prime}\left(\mathrm{x}_{2}\right)=\alpha\left(\mathrm{m}_{2}\right)$, then, $\left(\mathrm{x}_{1}, \mathrm{x}_{2}\right) \in \operatorname{Ker}\left(\alpha^{\prime}\right)=\operatorname{Ker}(\alpha) \subseteq \operatorname{Ker}(\beta)=\operatorname{Ker}\left(\beta^{\prime}\right)$. So, $\beta^{\prime}\left(\mathrm{x}_{1}\right)=$ $\beta^{\prime}\left(x_{2}\right)$. Since $M_{S}$ is C-M-P-injective, so there exists an S-homomorphismo: $M_{S} \rightarrow M_{S}$ such that $\sigma i_{1}=$ $\mathrm{i}_{2} \varphi$, then $\sigma \mathrm{i}_{1} \alpha^{\prime}=\mathrm{i}_{2} \varphi \alpha^{\prime}$.This implies that $\sigma \mathrm{i}_{1} \alpha^{\prime}=\mathrm{i}_{2} \beta^{\prime}$, then $\beta=\sigma \alpha$.Therefore, $\beta \in \mathrm{T} \alpha$ and $\ell_{\mathrm{T}}(\operatorname{Ker} \alpha) \subseteq$ $\mathrm{T} \alpha$. Then, we have $\mathrm{T} \alpha=\ell_{\mathrm{T}}(\operatorname{Ker} \alpha)$.

$(2 \rightarrow 3)$ Let $\mathrm{f}: \alpha(\mathrm{M}) \rightarrow \mathrm{M}_{\mathrm{S}}$ be a homomorphism, where $\alpha$ is a closed homomorphism. Since Ker $\alpha \subseteq$ Kerf $\alpha$,then $\ell_{\mathrm{T}}(\operatorname{Kerf} \alpha) \subseteq \ell_{\mathrm{T}}(\operatorname{Ker} \alpha)$. By (2), we have $\ell_{\mathrm{T}}(\operatorname{Kerf} \alpha) \subseteq \ell_{\mathrm{T}}(\operatorname{Ker} \alpha)=\mathrm{T} \alpha$ and so $\alpha \alpha \in \mathrm{T} \alpha$. $(3 \rightarrow 4)$ Let $\alpha, \beta$ and $\sigma \in \mathrm{T}$, where $\alpha$ is a closed homomorphism. Suppose that Ker $\alpha \subseteq \operatorname{Ker} \beta \subseteq \operatorname{Ker} \sigma \beta$. Then, by the homomorphism theorem 4.21 in ([2], P.53), there exists unique homomorphism $\mathrm{f}: \alpha(\mathrm{M}) \rightarrow \mathrm{M}_{\mathrm{S}}$ such that $\sigma \beta=f \alpha$. By using (3), f $\alpha \in \mathrm{T} \alpha$, then $\sigma \beta \in \mathrm{T} \alpha$. Therefore, we get $\mathrm{T} \beta \subseteq \mathrm{T} \alpha$.

$(4 \rightarrow 5)$ Let $\sigma \in \ell_{\mathrm{T}}\left[\beta\left(\mathrm{M}_{\mathrm{S}}\right) \times \beta\left(\mathrm{M}_{\mathrm{S}}\right) \cap \mathrm{Ker} \alpha\right]$ and $\alpha \beta$ is a closed homomorphism. We claim that $\operatorname{Ker} \alpha \beta \subseteq \operatorname{Ker} \sigma \beta$. For this, let $\left(\mathrm{m}_{1}, \mathrm{~m}_{2}\right) \in \operatorname{Ker} \alpha \beta$, so $\alpha \beta\left(\mathrm{m}_{1}\right)=\alpha \beta\left(\mathrm{m}_{2}\right)$. This implies that $\left(\beta\left(\mathrm{m}_{1}\right)\right.$, $\left.\beta\left(\mathrm{m}_{2}\right)\right) \in\left[\beta\left(\mathrm{M}_{\mathrm{s}}\right) \times \beta\left(\mathrm{M}_{\mathrm{s}}\right) \cap\right.$ Ker $\left.\alpha\right]$, then $\sigma \beta\left(\mathrm{m}_{1}\right)=\sigma \beta\left(\mathrm{m}_{2}\right)$. Thus $\left(\mathrm{m}_{1}, \mathrm{~m}_{2}\right) \in \operatorname{ker} \sigma \beta$. By (4), we have $\mathrm{T} \sigma \beta \subseteq \mathrm{T} \alpha \beta$ and $\sigma \beta=\mathrm{u} \alpha \beta$ for some $\mathrm{u} \in \mathrm{T}$. Therefore, this means that there is $\mathrm{u} \in \mathrm{T}$ such that $\sigma \beta=\mathrm{u} \alpha \beta$ for each $\alpha, \beta \in \mathrm{T}$. Since T is cancellative, so $\sigma=u \alpha$. Thus, $\sigma \in \ell_{\mathrm{T}}\left[\beta\left(\mathrm{M}_{\mathrm{s}}\right) \times \beta\left(\mathrm{M}_{\mathrm{s}}\right)\right.$. This means that $\sigma \in \ell_{\mathrm{T}}\left(\beta\left(\mathrm{M}_{\mathrm{S}}\right) \times \beta\left(\mathrm{M}_{\mathrm{S}}\right)\right) \cup \mathrm{T} \alpha \quad$ and $\quad \ell_{\mathrm{T}}\left[\beta\left(\mathrm{M}_{\mathrm{S}}\right) \times \beta\left(\mathrm{M}_{\mathrm{S}}\right) \cap \mathrm{Ker} \alpha\right] \quad \subseteq \quad \ell_{\mathrm{T}}\left(\beta\left(\mathrm{M}_{\mathrm{s}}\right) \times\right.$ $\left.\beta\left(\mathrm{M}_{\mathrm{s}}\right)\right) \cup \mathrm{T} \alpha$.Conversely, let $\sigma \in \ell_{\mathrm{T}}\left(\beta\left(\mathrm{M}_{\mathrm{s}}\right) \times \beta\left(\mathrm{M}_{\mathrm{s}}\right)\right) \cup \mathrm{T} \alpha$, hence this means that $\sigma \in \ell_{\mathrm{T}}\left(\beta\left(\mathrm{M}_{\mathrm{s}}\right) \times\right.$ $\beta\left(\mathrm{M}_{\mathrm{s}}\right)$ ) or $\sigma=\mathrm{u} \alpha$ for some $\mathrm{u} \in \mathrm{T}$. If $\sigma \in \ell_{\mathrm{T}}\left[\beta\left(\mathrm{M}_{\mathrm{s}}\right) \times \beta\left(\mathrm{M}_{\mathrm{s}}\right)\right]$, this means that $\sigma \beta\left(\mathrm{m}_{1}\right)=\sigma \beta\left(\mathrm{m}_{2}\right), \forall$ $\mathrm{m}_{1}, \mathrm{~m}_{2} \in \mathrm{M}_{\mathrm{S}}$. Now, for each $\mathrm{m}_{1}$ and $\mathrm{m}_{2} \in \mathrm{M}_{\mathrm{S}}$, we have $\left(\mathrm{m}_{1}, \mathrm{~m}_{2}\right) \in\left[\operatorname{Ker} \alpha \cap \beta\left(\mathrm{M}_{\mathrm{S}}\right) \times \beta\left(\mathrm{M}_{\mathrm{S}}\right)\right]$, which implies that $\alpha\left(\mathrm{m}_{1}\right)=\alpha\left(\mathrm{m}_{2}\right)$ and $\beta\left(\mathrm{m}_{1}\right)=\beta\left(\mathrm{m}_{2}\right)$. Since $\mathrm{u}$ is well-defined, so $\mathrm{u} \alpha\left(\mathrm{m}_{1}\right)=\mathrm{u} \alpha\left(\mathrm{m}_{2}\right)$. If $\sigma=\mathrm{u} \alpha$, then this implies that $\sigma\left(\mathrm{m}_{1}\right)=\sigma\left(\mathrm{m}_{2}\right)$. Thus, $\sigma \in \ell_{\mathrm{T}}\left(\beta\left(\mathrm{M}_{\mathrm{s}}\right) \times \beta\left(\mathrm{M}_{\mathrm{s}}\right) \cap \operatorname{Ker} \alpha\right)$. If $\sigma \in \ell_{\mathrm{T}}\left[\beta\left(\mathrm{M}_{\mathrm{s}}\right) \times\right.$ $\beta\left(\mathrm{M}_{\mathrm{s}}\right)$, then $\sigma \beta\left(\mathrm{m}_{1}\right)=\sigma \beta\left(\mathrm{m}_{2}\right)$. Hence, $\sigma \in \ell_{\mathrm{T}}\left(\beta\left(\mathrm{M}_{\mathrm{s}}\right) \times \beta\left(\mathrm{M}_{\mathrm{s}}\right) \cap \mathrm{Ker} \alpha\right)$ and $\ell_{\mathrm{T}}\left(\beta\left(\mathrm{M}_{\mathrm{s}}\right) \times \beta\left(\mathrm{M}_{\mathrm{s}}\right)\right) \cup \mathrm{T} \alpha$ $\subseteq \ell_{\mathrm{T}}\left[\beta\left(\mathrm{M}_{\mathrm{s}}\right) \times \beta\left(\mathrm{M}_{\mathrm{s}}\right) \cap\right.$ Ker $\left.\alpha\right]$. 
(5 $\rightarrow 1$ ) By taking $\beta=\mathrm{I}_{\mathrm{M}}$, we identified the map of $\mathrm{M}_{\mathrm{S}}$ in (5) and obtained that $\ell_{\mathrm{T}}($ ker $\alpha)=\mathrm{T} \alpha$ for every closed homomorphism $\alpha \in \mathrm{T}$. Now, let $\mathrm{N}$ be a closed M-cyclic subact of $\mathrm{S}$-act $\mathrm{M}_{\mathrm{S}}, \mathrm{so} \mathrm{N}=\alpha$ (M) for some closed homomorphism $\alpha \in T$. Assume that $i_{1}: \alpha(M) \rightarrow M_{S}$ is the inclusion map and $\alpha_{1}$ : $\mathrm{M}_{\mathrm{S}} \rightarrow \alpha(\mathrm{M})$ is a homomorphism induced by closed homomorphism $\alpha$. Let $\varphi$ be S-homomorphism from $\mathrm{N}$ into $\mathrm{M}_{\mathrm{S}}$. It is clear that $\varphi \alpha_{1}$ is $\mathrm{S}$-endomorphism of $\mathrm{M}_{\mathrm{S}}$. Since $\operatorname{Ker} \alpha=\operatorname{Ker} \alpha_{1} \subseteq \operatorname{Ker} \varphi \alpha_{1}$, whence for each $(\mathrm{x}, \mathrm{y}) \in \operatorname{Ker} \alpha$ implies $\alpha(\mathrm{x})=\alpha(\mathrm{y})$, and since $\varphi$ is well-defined, so $\varphi(\alpha(\mathrm{x}))=\varphi(\alpha(\mathrm{y}))$. Thus, we obtain $(\mathrm{x}, \mathrm{y}) \in \operatorname{Ker} \varphi$. This implies that $\ell_{\mathrm{T}}(\operatorname{ker} \varphi \alpha) \subseteq \ell_{\mathrm{T}}(\operatorname{ker} \alpha)$. Because $\ell_{\mathrm{T}}(\operatorname{ker} \alpha)=\mathrm{T} \alpha$, then we have T $\varphi \alpha \subseteq \mathrm{T} \alpha$. Thus, $\varphi \alpha \in \mathrm{T} \alpha$ and then there exists $\sigma \in \mathrm{T}$ such that $\varphi \alpha=\sigma \alpha$. Therefore $\mathrm{M}_{\mathrm{S}}$ is a C-M-P-injective act.

We can define a closed ideal as follows: an ideal of a monoid $\mathrm{S}$ is called a closed ideal if there is no proper essential extension (i.e. no proper $\cap$-large) inside $S$.

The next corollary characterizes the closed self principally injective monoids.

By Definition 2.3, a monoid $\mathrm{S}$ is called closed self principally injective monoid (for short, C-self-P-injective) in case that $\mathrm{S}_{\mathrm{S}}$ is closed quasi principally injective. Thus, the proof of the next corollary is clear by Theorem 2.6 and, hence, it is omitted.

Corollary 2.7: The following conditions are equivalent for a monoid S:

(1) $\mathrm{S}$ is C-self-P-injective.

(2) $\ell_{\mathrm{S}}\left(\gamma_{\mathrm{S}}(\mathrm{a})\right)=\mathrm{Sa}, \forall \mathrm{a} \in \mathrm{S}$, and closed right ideal aS.

(3) If $\mathrm{f:} \mathrm{aS} \rightarrow \mathrm{S}$ is $\mathrm{S}$-homomorphism, then $\mathrm{f}$ (a) $\in \mathrm{Sa}$ for all $\mathrm{a} \in \mathrm{S}$ and closed right ideal aS.

(4) $\gamma_{\mathrm{S}}(\mathrm{b}) \subseteq \gamma_{\mathrm{S}}(\mathrm{a})$, which implies that $\mathrm{Sa} \subseteq \mathrm{Sb}$ for any $\mathrm{a}, \mathrm{b} \in \mathrm{S}$, where $\mathrm{bS}$ is closed right ideal.

(5) $\ell_{\mathrm{S}}\left(\mathrm{bS} \cap\left(\gamma_{\mathrm{S}}(\mathrm{a}) \times \gamma_{\mathrm{S}}(\mathrm{a})\right)\right)=\ell_{\mathrm{S}}(\mathrm{bS} \times \mathrm{bS}) \cup \mathrm{Sa}, \forall \mathrm{a}, \mathrm{b} \in \mathrm{S}$.

In the subsequent theorem, we study some general properties of C-M-P-injective acts and C-self-P-injective monoids.

Theorem2.8: Let $\mathrm{M}_{\mathrm{S}}$ be C-M-P-injective $\mathrm{S}$-act and $\alpha, \beta \in \mathrm{T}=\mathrm{End}(\mathrm{M})$, where $\alpha$ is a closed homomorphism. Then, the following statements hold:

(1) If $\mathrm{f:} \alpha(\mathrm{M}) \rightarrow \beta(\mathrm{M})$ is a monomorphism (otherwise, an epimorphism), then there exists a T-epimorphism (otherwise, a T- monomorphism) $\sigma: \mathrm{T} \beta \rightarrow \mathrm{T} \alpha$.

(2) If $\alpha(M) \cong \beta(M)$, then $T \alpha \cong T \beta$.

Proof: (1) Let $\mathrm{f:} \alpha(\mathrm{M}) \rightarrow \beta(\mathrm{M})$ be an S-monomorphism, where $\mathrm{M}_{\mathrm{S}}$ is an S-act. Let $\mathrm{i}_{1}$ (otherwise, $\mathrm{i}_{2}$ ) be the inclusion maps of $\alpha(M)$ (otherwise, $\beta(M)$ ) into $M_{S}$. Then $f \alpha(m)=\beta(m)$ for all $m \in M_{S}$. Since $M_{S}$ is C-M-P-injective and $\alpha(M)$ is closed M-cyclic subact of $M_{S}$, so the S-homomorphism $i_{2}$ of can be extended to the S-homomorphism $\overline{\mathrm{f}}: \mathrm{M}_{S} \rightarrow \mathrm{M}_{S}$, such that $\overline{\mathrm{f}} \circ \mathrm{i}_{1}=\mathrm{i}_{2} \circ \mathrm{f}$. This means that $\overline{\mathrm{f}} \mathrm{i}_{1} \alpha(\mathrm{m})=$ $\mathrm{i}_{2} \mathrm{f} \alpha(\mathrm{m})$ for all $\mathrm{m} \in \mathrm{M}_{\mathrm{s}}$. Therefore, $\beta(\mathrm{m})=\overline{\mathrm{f}} \alpha(\mathrm{m})$ for all $\mathrm{m} \in \mathrm{M}_{\mathrm{s}}$. Define $\sigma: \mathrm{T} \beta \rightarrow \mathrm{T} \alpha$ by $\sigma(\lambda \beta)$ $=\lambda \overline{\mathrm{f}} \alpha, \lambda \beta \in \mathrm{T} \beta$. If $\lambda_{1} \beta=\lambda_{2} \beta$ for $\mathrm{m} \in \mathrm{M}_{\mathrm{S}}$, then $\overline{\mathrm{f}} \alpha(\mathrm{m})=\left(\overline{\mathrm{f}} \circ \mathrm{i}_{1}\right)(\alpha(\mathrm{m}))=\left(\mathrm{i}_{2} \circ \mathrm{f}\right)(\alpha(\mathrm{m}))=\mathrm{f}(\alpha(\mathrm{m}))$, and hence $\lambda \overline{\mathrm{f}} \alpha(\mathrm{m})=\lambda \mathrm{f}(\alpha(\mathrm{m}))$. Thus, $\sigma$ is well-defined. It is clear that $\sigma$ is T-homomorphism; in fact, let $\lambda \beta \in \mathrm{T} \beta$ and $\mathrm{g} \in \mathrm{T}$, then $\sigma(\mathrm{g}(\lambda \beta))=\sigma((\mathrm{g} \lambda) \beta)=\mathrm{g} \lambda \overline{\mathrm{f}} \alpha=\mathrm{g}(\lambda \overline{\mathrm{f}} \alpha)=\mathrm{g} \sigma(\lambda \beta)$. We claim that Ker $(\overline{\mathrm{f}} \alpha) \subseteq \operatorname{Ker} \alpha$. Let $\left(\mathrm{x}_{1}, \mathrm{x}_{2}\right) \in \operatorname{Ker}(\overline{\mathrm{f}} \alpha)$, which implies that $\overline{\mathrm{f}} \alpha\left(\mathrm{x}_{1}\right)=\overline{\mathrm{f}} \alpha\left(\mathrm{x}_{2}\right)$. This implies that $\mathrm{f}\left(\alpha\left(\mathrm{x}_{1}\right)\right)=\mathrm{f}$ $\left(\alpha\left(\mathrm{x}_{2}\right)\right)$. Since $\mathrm{f}$ is monomorphism, so $\alpha\left(\mathrm{x}_{1}\right)=\alpha\left(\mathrm{x}_{2}\right)$. Thus, $\left(\mathrm{x}_{1}, \mathrm{x}_{2}\right) \in \operatorname{Ker} \alpha$. By theorem3.4(4), we have $\mathrm{T} \alpha \subseteq \mathrm{T} \overline{\mathrm{f}} \alpha$, so there exists $\lambda \in \mathrm{T}$ such that $\alpha=\lambda \overline{\mathrm{f}} \alpha$, then $\alpha=\lambda \overline{\mathrm{f}} \alpha=\sigma(\lambda \beta) \in \sigma(\mathrm{T} \beta)$. This implies that $\mathrm{T} \alpha=\sigma(\mathrm{T} \beta)$. Then, $\sigma$ is T-epimorphism.

For the second part (i.e. If $\mathrm{f}: \alpha(\mathrm{M}) \rightarrow \beta(\mathrm{M})$ is epimorphism, then there exists a T- monomorphism $\sigma: \mathrm{T} \beta \rightarrow \mathrm{T} \alpha)$, as in above. Let $\mathrm{f:} \alpha(\mathrm{M}) \rightarrow \beta(\mathrm{M})$, and by assumption, $\mathrm{f}$ is $\mathrm{S}$-epimorphism. Since $\mathrm{M}_{\mathrm{S}}$ is C-M-P-injective, so $i_{2}$ of can be extended to $\bar{f}: M_{S} \rightarrow M_{S}$ such that foi $i_{1}=i_{2}$ of, where $i_{1}$ and $i_{2}$ are 
the inclusion map of $\alpha(M)$ into $M_{S}$ and $\beta(M)$ into $M_{S}$, respectively. Define $\sigma: \mathrm{T} \beta \rightarrow$ T $\alpha$ by $\sigma(\lambda \beta)$ $=\lambda \overline{\mathrm{f}} \alpha$, for $\lambda \in \mathrm{T}$. As in the first part of the proof, $\sigma$ is well-defined, then $\lambda_{1} \overline{\mathrm{f}} \alpha=\lambda_{2} \overline{\mathrm{f}} \alpha$. Since $\overline{\mathrm{f}} \alpha(\mathrm{M})=$ $\bar{f}_{0} i_{1}(\alpha(M))=i_{2} \mathrm{of}(\alpha(M))=f \alpha(M)=\beta(M)$, then $\lambda \bar{f} \alpha(M)=\lambda \beta(M)$, hence $\lambda_{1} \beta(M)=\lambda_{1} \bar{f} \alpha(M)=\lambda_{2} \bar{f} \alpha(M)=$ $\lambda_{2} \beta(\mathrm{M})$, then $\lambda_{1} \beta=\lambda_{2} \beta$. Hence $\sigma$ is T-monomorphism.

(2) By using (1).

Before describing the next corollary, we need the following definition:

Definition 2.9: [2, P.20] Let $S$ be a semigroup. A nonempty subset $K$ of $S$ is called left ideal of $S$ if $\mathrm{SK} \subseteq \mathrm{K}$, a right ideal of $\mathrm{S}$ if $\mathrm{KS} \subseteq \mathrm{K}$, and an ideal of $\mathrm{S}$ if $\mathrm{SK} \subseteq \mathrm{K}$ and $\mathrm{KS} \subseteq \mathrm{K}$.

Recall that an ideal of a monoid $\mathrm{S}$ is called a closed ideal if there is no proper essential extension (i.e. no proper $\mathrm{C}$-large) inside $\mathrm{S}$.

In a similar way, we can define a closed right (otherwise, left) ideal of a monoid $\mathrm{S}$ if there is no proper essential extension right (otherwise, left) ideal (i.e. no proper $\mathrm{n}$-large right (otherwise, left) ideal) inside $\mathrm{S}$.

Corollary 2.10: Let $S$ be C-self-P-injective monoid. Then, for any $s, t \in S$, and closed right ideal $b S$, the following statements hold:

(1) If $\mathrm{f}: \mathrm{bS} \rightarrow \mathrm{aS}$ is monomorphism (otherwise, an epimorphism), then there exists an epimorphism (otherwise, a monomorphism) $\sigma: \mathrm{Sa} \rightarrow \mathrm{Sb}$.

(2) If $b S \cong a S$, then $S b \cong S a$.

The next proposition explains the concepts of Co-Hopfian and the directly finite that coincide under C- quasi principal injectivity condition.

Proposition 2.11: Every C- quasi principally injective act and directly finite is co-Hopfian.

Proof: Similar to the proof of proposition 2.17 in [1], by replacing $\mathrm{M}_{\mathrm{S}}$ being C-M-injective act by being C-QP-injective.

The following proposition shows that the concepts of Hopfian and co-Hopfian are coincided in-terms of C-QP-injective property.

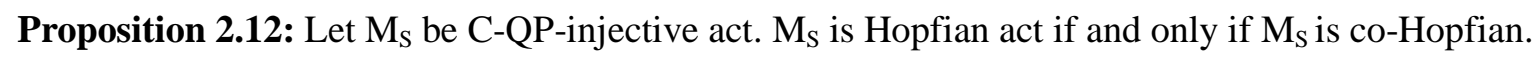

Proof: $\Rightarrow$ ) As every Hopfian is directly finite (For this, if for any $\alpha, \beta \in \operatorname{End}\left(M_{S}\right)$ and $\alpha o \beta=I$ ), where I is the identity endomorphism, then this means that $\alpha$ is surjective. Since $M_{S}$ is Hopfian, then $\alpha$ is an isomorphism and $\beta$ is the inverse of $\alpha$. Thus $\beta o \alpha=I$, which implies that $\mathrm{M}_{\mathrm{S}}$ is a directly finite act, so by Proposition 2.11, $\mathrm{M}_{\mathrm{S}}$ is co-Hopfian.

$\Leftarrow)$ Let $\mathrm{f}$ be surjective endomorphism of $\mathrm{M}_{\mathrm{S}}$, then the inclusion map i:f $(\mathrm{M}) \rightarrow \mathrm{M}_{\mathrm{S}}$ is isomorphism ( since $\mathrm{M}_{\mathrm{S}}$ is co-Hopfian). Thus foi $=\mathrm{I}_{\mathrm{f}(\mathrm{M})}$. By proposition $2.11, \mathrm{M}_{\mathrm{S}}$ is directly finite, so iof $=\mathrm{I}_{\mathrm{M}}$ ( since $\mathrm{f}$ $\left.(M) \cong M_{S}\right)$. Thus $f$ is injective and then it is isomorphism. Therefore, $M_{S}$ is Hopfian.

Recall that $A$ right $S$-act $B_{S}$ is a retract of a right $S$-act, as if and only if there exists a subact $W$ of $A_{S}$ and epimorphism $\mathrm{f}: A_{S} \rightarrow W$, such that $B_{S} \cong \mathrm{W}$ and $\mathrm{f}(\mathrm{x})=\mathrm{x}$ for every $\mathrm{x} \in \mathrm{W}$ ([2],P.84).

Proposition2.13: Let $\mathrm{M}_{\mathrm{S}}$ be $\mathrm{S}$-act and $\mathrm{N}$ be closed $\mathrm{M}$-cyclic subact of $\mathrm{M}_{\mathrm{S}}$.If $\mathrm{N}$ is C-M-P-injective, then $\mathrm{N}$ is a retract subact of $\mathrm{M}_{\mathrm{S}}$.

Proof: Let $\mathrm{i}_{\mathrm{N}}$ be the inclusion map of closed M-cyclic subact $\mathrm{N}$ of $\mathrm{S}$-act $\mathrm{M}_{\mathrm{S}}$. Since $\mathrm{N}$ is C-M-P-injective, then there exists an S-homomorphism g: $\mathrm{M}_{\mathrm{S}} \rightarrow \mathrm{N}$ such that go $\mathrm{i}_{\mathrm{N}}=\mathrm{I}_{\mathrm{N}}$, hence $\mathrm{i}_{\mathrm{N}}$ has left inverse and $\mathrm{i}(\mathrm{N})$ is a retract subact of $\mathrm{M}_{\mathrm{S}}$, but $\mathrm{N}=\mathrm{i}(\mathrm{N})$, so $\mathrm{N}$ is a retract subact of $\mathrm{M}_{\mathrm{S}}$.

By replacing the property of $\mathrm{M}_{\mathrm{S}}$ from C-quasi injective act to C-M-P-injective act in proposition 2.5 in [1], we can proof the following Proposition:

Proposition 2.14: Let $\mathrm{M}_{\mathrm{S}}$ be a C-M-P-injective act. Then every fully invariant closed subact of $\mathrm{M}_{\mathrm{S}}$ is C-quasi principally injective. 
Proposition 2.15: Every retract subact of C-M-P-injective is C-M-P-injective.

Proof: Assume that $\mathrm{N}$ is C-M-P-injective S-act and A is a retract subact of N. Let X be closed $\mathrm{M}$-cyclic subact of S-act $\mathrm{M}_{\mathrm{S}}$ and $\mathrm{f}$ be S-homomorphism from $\mathrm{X}$ into A. Since $\mathrm{N}$ is C-M-P-injective act, so there exists $S$-homomorphism $g$ from $M$ into $N_{S}$ such that $g \circ i_{X}=j_{A} \circ$, where $i_{X}$ is the inclusion map of $X$ into $M_{S}$ and $j_{A}$ is the injection map of $A$ into $N_{S}$. Put $h=\pi_{A} o g$, where $\pi_{A}$ is the projection map of $\mathrm{N}_{\mathrm{S}}$ onto $\mathrm{A}$, then $\mathrm{h} \circ \mathrm{i}_{\mathrm{X}}=\pi_{\mathrm{A}} \circ \mathrm{g} \circ \mathrm{i}_{\mathrm{X}}=\pi_{\mathrm{A}} \circ \mathrm{j}_{\mathrm{A}} \circ \mathrm{f}=\mathrm{f}$ and $\mathrm{A}$ is C-M-P-injective act.

Proposition 2.16: Let $M_{S}$ and $N_{S}$ are two S-acts. If $N_{S}$ is C-M-P-injective act, and $B_{S}$ is a closed M-cyclic subact of $\mathrm{M}_{\mathrm{S}}$, then $\mathrm{N}_{\mathrm{S}}$ is C-B-P-injective act.

Proof: Let X be closed B-cyclic subact of B. Since B is closed M-cyclic subact of $\mathrm{M}_{\mathrm{S}}$, so by lemma2.4 in [13], $\mathrm{X}$ is closed M-cyclic. Let $\mathrm{f}$ be $\mathrm{S}$-homomorphism from $\mathrm{X}$ into $\mathrm{N}_{\mathrm{S}}$. Since $\mathrm{N}_{\mathrm{S}}$ is C-M-P-injective act, so there exists $S$-homomorphism $g$ from $M_{S}$ into $N_{S}$ such that $g \circ i_{B} \circ i_{X}=f$, where $i_{X}, i_{B}$ be the inclusion map of $X$ into $B$ and $B$ into $M_{S}$, respectively. $P u t h=g \circ i_{B}$, then $h \circ i_{X}=g \circ i_{B} \circ i_{X}=f$. Thus $N_{S}$ is C-B-P-injective act.

Corollary 2.17: Let $M_{S}$ and $N_{S}$ be two S-acts. Then, $N_{S}$ is C-M-P-injective act if and only if $N_{S}$ is C-X-P-injective act for every closed $\mathrm{M}$-cyclic subact $\mathrm{X}$ of $\mathrm{M}_{\mathrm{S}}$.

Proof: Suppose that $\mathrm{N}_{\mathrm{S}}$ is C-M-P-injective act, then by proposition 2.16, we have $\mathrm{N}_{\mathrm{S}}$ is C-X-P-injective for every closed $\mathrm{M}$-cyclic subact $\mathrm{X}$ of $\mathrm{M}_{\mathrm{S}}$. The converse is clear.

Proposition 2.18: Let $M_{S}$ be an $S$-act and $\left\{N_{i} \mid i \in I\right\}$ be a family of $S$-acts. Then $\prod_{i \in I} N_{i}$ is C-M-P-injective act if and only if $\mathrm{N}_{\mathrm{i}}$ is C-M-P-injective act for every $\mathrm{i} \in \mathrm{I}$.

Proof: $\Rightarrow$ ) Assume that $\mathrm{N}_{S}=\prod_{i \in I} \mathrm{~N}_{\mathrm{i}}$ is C-M-P-injective. Let $\mathrm{X}$ be closed M-cyclic subact of S-act $\mathrm{M}_{\mathrm{S}}$ and $\mathrm{f}$ be $\mathrm{S}$-homomorphism from $\mathrm{X}$ to $\mathrm{N}_{\mathrm{i}}$. Since $\mathrm{N}_{\mathrm{S}}$ is C-M-P-injective act then there exists S-homomorphism g: $\mathrm{M}_{\mathrm{S}} \rightarrow \mathrm{N}_{\mathrm{S}}$ such that goi $\mathrm{i}_{\mathrm{X}}=\mathrm{j}_{\mathrm{i}}$ of, where $\mathrm{i}_{\mathrm{X}}$ is the inclusion map of $\mathrm{X}$ into $\mathrm{M}_{\mathrm{S}}$ and $\mathrm{j}_{\mathrm{i}}$ is the injection map of $N_{i}$ into $N_{S}$. Define $h: M_{S} \rightarrow N_{i}$ by $h=\pi_{i} \circ g$, where $\pi_{i}$ is the projection map of $N_{S}$ onto $N_{i}$. Then hoi $i_{X}=\pi_{i} \circ g \circ i_{X}=\pi_{i} \circ j_{i} \circ f=f$. That is, for all $x \in X, h(x)=h\left(i_{X}(x)\right)=\pi_{i}(g(x))=\pi_{i}\left(g\left(i_{X}(x)\right)\right)$ $=\pi_{\mathrm{i}}\left(\mathrm{j}_{\mathrm{i}}(\mathrm{f}(\mathrm{x}))\right)=\left(\pi_{\mathrm{i}} \circ \mathrm{j}_{\mathrm{i}}\right)(\mathrm{f}(\mathrm{x}))=\mathrm{f}(\mathrm{x})$.

$\Leftarrow$ ) Assume that $\mathrm{N}_{\mathrm{i}}$ is C-M-P-injective act for each $\mathrm{i} \in \mathrm{I}$, where $\mathrm{M}_{\mathrm{S}}$ is $\mathrm{S}$-act. Let $\mathrm{X}$ be closed M-cyclic subact of $\mathrm{M}_{\mathrm{S}}$ and $\mathrm{f}$ be $\mathrm{S}$-homomorphism from $\mathrm{X}$ to $\mathrm{N}_{\mathrm{S}}=\prod_{\mathrm{i} \in \mathrm{I}} \mathrm{N}_{\mathrm{i}}$. Since $\mathrm{N}_{\mathrm{i}}$ is C-M-P-injective act, then there exists S-homomorphism $\beta_{\mathrm{i}}: \mathrm{M}_{\mathrm{S}} \rightarrow \mathrm{N}_{\mathrm{i}}$, such that $\beta_{\mathrm{i}} \circ \mathrm{i}_{\mathrm{X}}=\pi_{\mathrm{i}}$ of, so there exists S-homomorphism $\beta$ : $M_{S} \rightarrow N_{S}$ such that $\beta=j_{i} \circ \beta_{i}$. We claim that $\beta \circ i_{X}=f$. Since $\beta \circ i_{X}=j_{i} \circ \beta_{i} \circ i_{X}=j_{i} \circ \pi_{i} \circ f=f$, so we obtain $\mathrm{f}=\beta \circ \mathrm{i}$. Therefore, $\mathrm{N}_{\mathrm{S}}$ is C-M-P-injective.

Corollary 2.19: Let $M_{S}$ and $N_{i}$ be $S$-acts, where $i \in I$ and $I$ is a finite index set. Then, for every $i, N_{i}$ is C-M-P-injective if and only if $\bigoplus_{\mathrm{i}=1}^{\mathrm{n}} \mathrm{N}_{\mathrm{i}}$ is C-M-P-injective.

The next theorem gives the relationship between injective and C-N-P-injective acts:

Theorem 2.20: The following statements are equivalent for $S$-act $M_{S}$ :

(1) $M_{S}$ is injective act,

(2) $\mathrm{M}_{\mathrm{S}}$ is C-N-P-injective act for every S-act $\mathrm{N}$.

Proof: $(1 \Longrightarrow 2)$ It is obvious.

$(2 \Longrightarrow 1)$ Assume that $\mathrm{M}_{\mathrm{S}}$ is C-N-P-injective act and $\mathrm{E}(\mathrm{M})$ is injective envelope of $\mathrm{M}_{\mathrm{S}}$. By corollary 2.19, $\mathrm{M}_{\mathrm{S}} \oplus \mathrm{E}(\mathrm{M})$ is C-N-P-injective. Put $\mathrm{N}_{\mathrm{S}}=\mathrm{M}_{\mathrm{S}} \oplus \mathrm{E}(\mathrm{M})$. Thus, $\mathrm{M}_{\mathrm{S}} \oplus \mathrm{E}(\mathrm{M})$ is C-M $\oplus \mathrm{E}-\mathrm{P}$-injective. By proposition 2.15, $\mathrm{M}_{\mathrm{S}}$ is $\mathrm{C}-\mathrm{M} \oplus \mathrm{E}-\mathrm{P}-$ injective act. Consider the inclusion map i: $\mathrm{M}_{\mathrm{S}} \rightarrow \mathrm{E}(\mathrm{M})$ and the injection maps $j_{1}: E(M) \rightarrow M_{S} \oplus E(M), j_{2}: M_{S} \rightarrow M_{S} \oplus E(M)$, and $I_{M}: M_{S} \rightarrow M_{S}$ are the identity maps of $M_{S}$. Let $\pi_{M}: M_{S} \oplus E(M) \rightarrow M_{S}$ be the projection map such that $\pi_{M} \circ j_{2}=I_{M}$. Now, $M_{S} \oplus E(M)$ is C-quasi injective, so this implies that there exists S-homomorphism g: $\mathrm{M}_{\mathrm{S}} \oplus \mathrm{E}(\mathrm{M}) \rightarrow \mathrm{M}_{\mathrm{S}} \oplus \mathrm{E}(\mathrm{M})$ such that $g \circ j_{1} \circ i=j_{2} \circ I_{M}$, then $\pi_{M} \circ g \circ j_{1} \circ i=\pi_{M} \circ j_{2} \circ I_{M}$. Thus $I_{M}=\pi_{M} \circ g \circ j_{1} \circ i$. Put $f=\pi_{M} \circ g \circ j_{1}$ and 
then $I_{M}=$ foi. Therefore, $M_{S}$ is a retract of $E(M)$ and then it is injective.

Definition 2.21: An S-act $M_{S}$ satisfies the CM-property if every closed subact of $M_{S}$ is an M-cyclic subact of $\mathrm{M}_{\mathrm{S}}$.

The following proposition provides a relationship among the extending act, C-M-injective, and C-M-P-injective:

Proposition 2.22: The following statements are equivalent for $S$-act $M_{S}$ :

(1) $\mathrm{M}_{\mathrm{S}}$ is an extending act,

(2) Every S-act is C-M-injective,

(3) Every S-act is C-M-P-injective and $\mathrm{M}_{\mathrm{S}}$ satisfies CM-property.

Proof: $(1 \Longrightarrow 2)$ It is obvious.

$(2 \Rightarrow 3)$ Let $\mathrm{N}$ be a closed subact of $\mathrm{S}$-act $\mathrm{M}_{\mathrm{S}}$. By using(2), $\mathrm{N}$ is C-M-injective act. Thus, by proposition 2.7, $\mathrm{N}$ is a retract subact of $\mathrm{M}_{\mathrm{S}}$ and hence every retract subact is M-cyclic, by remarks and examples 2.3(2) in [8]. Thus, $\mathrm{M}_{\mathrm{S}}$ satisfies the CM-property. The other part is obvious.

$(3 \Rightarrow 1)$ Let $N$ be any closed subact of $S$-act $M_{S}$. Since $M_{S}$ satisfies the CM-property, so N is M-cyclic. By using (3), $\mathrm{N}$ is C-M-P-injective act. By proposition 2.13, $\mathrm{N}$ is a retract subact of $\mathrm{M}_{\mathrm{S}}$. Thus $\mathrm{M}_{\mathrm{S}}$ is extending act.

Theorem 2.23: The following statements are equivalent for the projective act $\mathrm{M}_{\mathrm{S}}$ :

(1) Every homomorphic image of any C-M-P-injective act is C-M-P-injective.

(2) Every homomorphic image of any C-M-injective act is C-M-P-injective.

(3) Every homomorphic image of any M-injective act is C-M-P-injective.

(4) Every homomorphic image of any injective act is C-M-P-injective.

(5) Every closed M-cyclic subact of $\mathrm{M}_{\mathrm{S}}$ is projective.

Proof: $(1 \Longrightarrow 2),(2 \Longrightarrow 3)$ and $(3 \Longrightarrow 4)$ are obvious.

$\left(4 \Rightarrow 5\right.$ ) Let A be closed M-cyclic subact of $\mathrm{M}_{\mathrm{S}}$ and $\mathrm{f}$ be $\mathrm{S}$-epimorphism from $\mathrm{S}$-act $\mathrm{N}_{\mathrm{S}}$ onto $\mathrm{S}$-act $\mathrm{B}_{\mathrm{S}}$. Let $\mathrm{g}$ be $\mathrm{S}$-homomorphism from $\mathrm{A}$ into $\mathrm{B}_{\mathrm{S}}$. Since every act can be embedded into an injective act, by corollary 1.6 in ([2], P.186), thus $\mathrm{N}_{\mathrm{S}}$ embedded into $\mathrm{E}$ and $\mathrm{i}_{\mathrm{N}}$ is the inclusion map of $\mathrm{N}$ into E. Letr: $\mathrm{E} \rightarrow \mathrm{E} / \rho$ is the canonical projection map such that $\rho=$ Kerf. Define $\ell: \mathrm{B}_{\mathrm{S}} \rightarrow \mathrm{E} / \rho$ by $\ell(\mathrm{b})=[\mathrm{b}]_{\rho}$, for all $b \in B_{S}$, where $b=f(n)$ and $n \in N_{S}$. It is clear that $\ell$ is well-defined and an S-homomorphism. By using (4), E/ $\rho$ is C-M-P-injective, so $\ell \circ g$ extends to $S$-homomorphism $g^{*}$ from $M$ into $E / \rho$, such that $\mathrm{g}^{*} \circ \mathrm{i}_{\mathrm{A}}=\ell \circ \mathrm{g}$. Since $\mathrm{M}_{\mathrm{S}}$ is projective, so $\mathrm{g}^{*}$ can be lifted to $\mathrm{S}$-homomorphism $\mathrm{h}$ from $\mathrm{M}_{\mathrm{S}}$ into $\mathrm{E}$, such that $\pi \circ \mathrm{h}=\mathrm{g}^{*}$. Since $\mathrm{E}$ is injective by assumption, then $\mathrm{h}$ represents the extension of the S-homomorphism $\alpha$ from A into E. This means that ho $\mathrm{i}_{\mathrm{A}}=\alpha$. Leth*: $\mathrm{A} \rightarrow \mathrm{N}_{\mathrm{S}}$ is defined by $\mathrm{h}^{*}(\mathrm{a})=\alpha(\mathrm{a})$, for all a $\in$ A. Now, $\ell \circ g=g^{*} \circ i_{A}=\pi \circ h \circ i_{A}=\pi \circ \alpha=\pi \circ i_{N} \circ h^{*}=\ell \circ f \circ h^{*}$. Thus, fo $h^{*}=g$ (since $\ell$ is monomorphism) and $\mathrm{A}$ is projective act.

$(5 \Rightarrow 1)$ Let $\mathrm{N}_{\mathrm{S}}$ be C-M-P-injective act and $\mathrm{f}: \mathrm{N} \rightarrow \mathrm{W}_{\mathrm{S}}$ be $\mathrm{S}$-epimorphism. Let A be a closed M-cyclic subact of $S$-act $\mathrm{M}_{\mathrm{S}}$ and $\mathrm{g}$ be any $\mathrm{S}$-homomorphism from A into $\mathrm{W}_{\mathrm{S}}$. Now, since $\mathrm{A}$ is projective by using (5), so g can be lifted to S-homomorphism $h$ from A into $N_{S}$. Since $N_{S}$ is C-M-P-injective act, so $h$ extends to $S$-homomorphism $h^{*}$ from $M_{S}$ into $N_{S}$ (this means that $h=h^{*} \circ i_{A}$, where $i_{A}$ is the inclusion map of A into $M_{S}$ ). Put $g^{*}=f \circ h^{*}$. Now, $g^{*} \circ i_{A}=f \circ h^{*} \circ i_{A}=f \circ h=g$. Thus, $g^{*} \circ i_{A}=g$ and $\mathrm{W}_{\mathrm{S}}$ is C-M-P-injective act.

\section{Acknowledgements}

The first author would like to thank Mustansiriyah University (www.uomustansiriyah.edu.iq) Baghdad-Iraq for its support in the present work.

Conflicts of Interest: The authors declare no conflicts of interest in this paper. 


\section{References}

1. Shaymaa, A. A. Kareem and Ahmed, A. A. Kareem 2019. About the Closed Quasi Injective S-Acts Over Monoids, Pure and Applied Mathematics Journal. Special Issue: Algebra with Its Applications, 8(5): 88-92. doi: 10.11648/j.pamj.20190805.12

2. Kilp, M., Knauer, U. and Mikhalev, A.V. 2000. Monoids acts and categories with applications to wreath products and graphs, Walter de Gruyter. Berlin. New York.

3. Yan, T. 2011. Generalized injective S-acts on a monoid, Advances in mathematics, 40(4): 421-432.

4. Jupil, K. 2008. PI-S-systems, Journal of Chungcheong Mathematical Society, 21(4): 591-599.

5. Shaymaa, A. 2018. about the generalizations in acts over monoids, LAP LAMBERT Academic Publishing, Germany.

6. Shaymaa, A.2018. On Finitely Generated in S-acts over monoids, Noor Publishing, Germany.

7. Shaymaa, A. 2015. Generalizations of quasi injective $\mathrm{S}$-acts over monoids, $\mathrm{PhD}$. thesis, College of Science, University of Al-Mustansiriyah, Baghdad, Iraq.

8. Abbas, M.S. and Shaymaa, A. 2015. Quasi principally injective acts over monoids, Journal of advances in mathematics, 10(5): 3493-3502.

9. Abdul Kareem, S.A. 2020. Dual of Extending Acts. Iraqi Journal of Science, 64-71. https://doi.org/10.24996/ijs.2020.SI.1.9

10. Ahsan, J. 1987. Monoids characterized by their quasi injective S-acts, Semigroup forum, 36(3): 285-292.

11. Lopez, A. M., Jr. and Luedeman, J. K. 1979. Quasi-injective S-acts and their S-endomorphism Semigroup, Czechoslovak Math.J. , 29(104): 97-104.

12. Al-Bahrani B. H., Rahman M. Q. 2020. On y-closed Rickart Modules, Iraqi Journal of Science, 61(10): 2681-2686 .DOI: 10.24996/ijs.2020.61.10.25

13. Shaymaa, A. 2017. Extending and P-extending S-act over monoids, International Journal of Advanced Scientific and Technical Research, 2(7): 171-178. 